\section{KENTRON}

REVUE PLURIDISCIPLINAIR

DU MONDE ANTIQUE

\section{Kentron}

Revue pluridisciplinaire du monde antique

$23 \mid 2007$

La démesure (fin)

\title{
Pierre Sineux, Amphiaraos, guerrier, devin et guérisseur
}

\section{Michelle Lacore}

\section{(2) OpenEdition}

\section{Journals}

Édition électronique

URL : http://journals.openedition.org/kentron/1759

DOI : 10.4000/kentron.1759

ISSN : 2264-1459

Éditeur

Presses universitaires de Caen

\section{Édition imprimée}

Date de publication : 31 décembre 2007

Pagination : 197-198

ISBN : 978-2-84133-321-9

ISSN : 0765-0590

\section{Référence électronique}

Michelle Lacore, « Pierre Sineux, Amphiaraos, guerrier, devin et guérisseur », Kentron [En ligne], 23 | 2007 , mis en ligne le 16 mars 2018, consulté le 19 novembre 2020. URL : http://journals.openedition.org/ kentron/1759; DOI : https://doi.org/10.4000/kentron.1759

\section{(c) (i) (3)}

Kentron is licensed under a Creative Commons Attribution-NonCommercial-NoDerivatives 3.0 International License. 
d'accéder au nomos politique. L'importance politique de la figure de l'orateur athénien Lycurgue n'est pas contestable, mais P. Brun montre que le titre de législateur, qui lui est donné par la tradition, procède d'une stratégie familiale d'amplification et n'est en rien corroboré par les inscriptions.

À côté de la stricte application de la loi, même adoucie par une perspective casuistique, se fait jour, tout au long de cette très longue histoire, l'exigence d'équité qui s'appuie sur la jurisprudence mais l'inspire aussi, et nous en confirmerions volontiers l'importance par un texte sophistique très ancien, l'Anonyme de Jamblique, qui loue sans réserve le droit, dût-il amener parfois à accepter un désavantage personnel.

Michelle LACORE

\section{Pierre Sineux, Amphiaraos, guerrier, devin et guérisseur, Paris, Les Belles Lettres (Vérité des Mythes), 2007, 276 p.}

L'ouvrage érudit de P. Sineux représente un considérable apport, comblant une surprenante lacune dans le domaine de l'histoire des cultes grecs. En effet, l'étude pénétrante de P. Vicaire, «Images d'Amphiaraos dans la Grèce archaïque et classique» (BAGB 1979), s'était cantonnée aux sources poétiques, envisagées du point de vue de l'élaboration progressive d'un «personnage». À ce point de vue, P. Sineux, à la suite de J.-P. Vernant, substitue la notion d'une «figure » qui s'est actualisée au cours du V $V^{e}$ siècle athénien. Pour dessiner les contours de cette figure, et traiter du développement de son culte, l'auteur s'appuie sur tous les types de sources à la disposition de l'historien.

Le livre étudie successivement, en six chapitres, les données poétiques du mythe du guerrier devin, ensuite la migration du culte d'Amphiaraos, devenu dieu, de Thèbes à Orôpos, près d'une source, lieu supposé de son anodos, éclairant ensuite les enjeux politiques pour les cités de Thèbes, d'Argos et d'Athènes, de la localisation définitive du sanctuaire aux marches de la Béotie et de l'Attique, pour aborder, dans la seconde moitié, le culte lui-même de cette divinité à la double fonction oraculaire et guérisseuse; l'évocation des rites préparatoires à la consultation (purification par le jeûne, sacrifices, offrandes) est suivie d'un chapitre consacré à l'étude du rite essentiel de l'incubation pratiqué dans l'Amphiaraion d'Orôpos; ce rite, en dépit du caractère original de la persistance - affaiblie toutefois - de la fonction oraculaire, en rapport avec les affinités apolliniennes d'Amphiaraos, permet de rapprocher les figures divines d'Asclépios et d'Amphiaraos dans le dernier chapitre "Rêves, images et récits, oracles et guérisons ».

Leétude couvre la totalité du développement de ce culte, marqué par sa localisation thébaine à l'époque archaïque, puis par la translation définitive du sanctuaire, de Cnopia à Orôpos, à l'époque classique, avec une remarquable permanence jusqu'à la fin du paganisme, résistant à tous les changements de statut politique aux différentes époques. 
Cet ouvrage, muni d'une très riche bibliographie (p. 221-244) et d'un recueil de «figures» diverses (carte, plan du site archéologique, reproductions de vases, de stèles et d'ex-votos curieux), mobilise de façon exhaustive tant les ressources textuelles - attestations poétiques du mythe et du culte, d'Homère et de la Thébaïde à l'Amphiaraos d'Aristophane et à l'Hypsipyle d'Euripide, témoignages historiques des diverses périodes (Hérodote, Plutarque, Pausanias), éloquence politique (Hypéride) - que les sources épigraphiques et les vestiges archéologiques. Toutes ces sources sont l'objet d'un examen impartial et minutieux, qui procède par approches successives, particulièrement pour les inscriptions, ainsi IG, VII, 235 (étudiée p. 82, pour la qualité de dieu reconnue à Amphiaraos, puis p. 140 pour la définition des rites sacrificiels, p. 149-150 pour la taxe) ainsi que la très intéressante inscription d'affranchissement SEG, XV, 293 (p. 199), dont la formule de dédicace nous paraît reposer sur le participe apposé grapsanta gouvernant le relatif « conformément à ce que le dieu lui avait prescrit de graver sur une stèle avant $\mathrm{de}<\mathrm{la}>$ consacrer près de l'autel ». Nous avons particulièrement apprécié la discussion (p. 97-117) qui établit comme probable la fondation par les Athéniens de l'Amphiaraion d'Orôpos dans les années qui suivent la fin de la guerre d'Archidamos et la confrontation entre les indications de Pausanias, les stèles conservées et l'inscription IG, VII, 235 pour la détermination des sacrifices, ainsi que l'examen des indications des inscriptions relatives à l'éparché IG, VII, 235 et SEG, XXII, 370, p. 148-158.

L'intérêt approfondi porté dans cette étude d'historien aux sources poétiques les plus variées (épiques, lyriques, tragiques, comiques) mérite d'être salué: un petit regret cependant pour l'Hypsipyle. Certes les fragments de cette tragédie perdue sont étudiés dans le premier chapitre, mais l'évolution d'Amphiaraos vers un statut de dieu guérisseur et sauveur pourrait, selon nous, être très directement mise en rapport avec la figure euripidéenne du devin à la parfaite rectitude, qui sauve une esclave sans défense injustement accusée. La date de la tragédie - 410 probablement, selon les commentateurs, dont, tout récemment, F. Jouan dans son édition des Fragments d'Euripide, $3^{\mathrm{e}}$ volume CUF, 2002 - pourrait venir à l'appui de la période retenue pour la fondation de l'Amphiaraion. Plus d'une fois, le tragique a choisi des thèmes mythiques en rapport avec l'actualité cultuelle (voir l'Érechthée par exemple).

Il s'agit là cependant de données plus élusives que celles qui résultent de l'étude des vestiges épigraphiques.

Au total, ce livre est une somme sur un culte qui, pour n'être pas un culte politiquement majeur, est certainement l'un des plus attachants et des plus révélateurs des imbrications du politique et du religieux, manifestes même à propos d'un culte et d'un sanctuaire où la consultation individuelle est prépondérante.

Michelle LACORE 